\title{
Applications of Artificial Intelligence (AI) in healthcare: A review
}

\author{
Mohammed Yousef Shaheen
}

\begin{abstract}
Artificial intelligence is revolutionizing - and strengthening - modern healthcare through technologies that can predict, grasp, learn, and act, whether it's employed to identify new relationships between genetic codes or to control surgery-assisting robots. It can detect minor patterns that humans would completely overlook. This study explores and discusses the various modern applications of $\mathrm{Al}$ in the health sector. Particularly, the study focuses on three most emerging areas of Al-powered healthcare: Al-led drug discovery, clinical trials, and patient care. The findings suggest that pharmaceutical firms have benefited from $\mathrm{Al}$ in healthcare by speeding up their drug discovery process and automating target identification. Artificial Intelligence (Al) can help also to eliminate time-consuming data monitoring methods. The findings also indicate that Al-assisted clinical trials are capable of handling massive volumes of data and producing highly accurate results. Medical Al companies develop systems that assist patients at every level. Patients' medical data is also analyzed by clinical intelligence, which provides insights to assist them improve their quality of life.

Keywords: Artificial intelligence, Al-led drug discovery, Al-assisted clinical trials, patients' care.

\section{Introduction}

The healthcare industry is in the midst of a transformation. The causes of this revolution are rising total health-care cost and a growing lack of health-care experts. As a result, the healthcare industry is looking to implement new information technology-based solutions and processes that can cut costs and give solutions to these rising difficulties.
\end{abstract}


Healthcare systems around the world face huge issues, including a lack of access, high costs, waste, and an older population. Pandemics like the coronavirus (COVID-19) put a strain on healthcare systems, resulting in a lack of protective equipment, insufficient or erroneous diagnostic tests, overworked physicians, and a lack of information exchange, to mention a few consequences (Greenberg et al., 2020) (Pavli et al., 2021). More crucially, a healthcare catastrophe like COVID-19 or the development of the human immunodeficiency virus (HIV) in the 1980s exposes the stark reality of our health-care systems' flaws. As healthcare crises exacerbate current difficulties, we can reinvent and actualize systems of care and backoffice health systems, such as: Inequitable healthcare access, that there are not enough on-demand healthcare services, high costs, a lack of price transparency (Maphumulo \& Bhengu, 2019). Technological breakthroughs are being adopted slowly. Burnout among healthcare practitioners is a result of physicians' incapacity to keep up with the latest breakthroughs in medicine due to the large amount of data to be assimilated.

As we focus on these issues, we should keep in mind that they are interconnected, providing the impression that healthcare is difficult when, in fact, it is given through complex systems. That isn't to suggest that providing outstanding healthcare is not difficult; nevertheless, we can create systems with less complication, resulting in better care and a system that works for everyone. AI should be a critical enabler of healthcare simplification and the development of intelligent care systems. The COVID-19 problem demonstrates how AI may be used for a variety of purposes, including diagnoses and treatment decision assistance, as well as contact tracing and the deployment of AI-driven technologies (van der Schaar et al., 2021) (Habermann, 2021) (Vaishya et al., 2020).

Each doctor's accomplishments and failures must be learned via experience before becoming part of the standard of care and best practices. Doctors gain knowledge from other doctors, research studies, medication and device businesses that promote goods, and their own triumphs and mistakes with their own patients. Each doctor's error are be identified and corrected, often at the expense of their patients(McNeill \& Walton, 2002). This form of learning represents human nature, and physicians are not immune to our brains' and learning systems' hard-wiring.

The issue is that the provider's prejudice and limits are a result of this anecdotal experience (Ross et al., 2009). In reality, based on their own personal observation, some physicians may mistakenly deceive themselves into believing that a diagnosis is correct or that a therapy is effective, despite the fact that it is counter to evidence backed by studies or the results of thousands of patients (McNeill \& Walton, 2002) (Shaheen, 2021a). Sometimes a clinician is just uninformed of new therapeutic care paths or better diagnostic modalities as a result of studies and data. To optimize reimbursement, clinicians must see as many patients as possible in the present medical environment. This leaves clinicians with little time to focus on secondary patient care chores, let alone stay current on medical breakthroughs. Doctors, on the other hand, now have immediate access to the insights and best practices of hundreds of cohorts, and they don't have to wait for best practices to be formalized into national standards of care (Ting et 
al., 2018). We can modify this calculus even more and act at a faster scale using AI than a particular physician or institution could (Holzinger et al., 2017).

Health professionals use the expertise of large numbers of clinical studies, the lessons of large numbers of patient treatment routes, and the cumulative experience of thousands of clinicians because they didn't have it at their fingertips (Shaheen, 2021a). This necessitates the use of technology, specifically artificial intelligence (AI) (Mayorga-Ruiz et al., 2019). Clinicians are vulnerable to cognitive and cultural biases as humans, but by offering a technological balancer in the knowledge base of providers, we can reduce, if not eradicate, the effects of such biases in $\mathrm{AI}$ (Woo, 2019).

\section{Applications of AI in Healthcare}

The following are some applications of medical artificial intelligence applications utilized in the healthcare industry:

\subsection{AI for Drug Discovery}

AI technology in healthcare has helped pharmaceutical companies speed up their drug discovery process. It, on the other hand, automates the identification of targets. In addition, by analyzing off-target compounds, AI in healthcare 2021 aids in drug repurposing (Díaz et al., 2019). As a result, in the AI and healthcare industries, AI drug discovery streamlines the process and reduces repeated work. (Chan et al., 2019).

Several remedies discovered by the leading biopharmaceutical companies are available. Pfizer is utilizing IBM Watson, a machine learning-based system, to help it find immuno-oncology treatments (P. Agrawal, 2018). Sanofi has agreed to employ Exscientia's artificial-intelligence (AI) platform to seek for metabolic-disease medications, while Roche subsidiary Genentech is relying on an Artificial intelligence system from GNS Healthcare in Cambridge, Massachusetts, to aid in its search for cancer treatments. Almost every major biopharmaceutical company has comparable collaborations or internal programs.

If proponents of these strategies are correct, AI and machine learning will bring in a new era of drug development that is faster, cheaper, and more effective. Some are skeptical, but most experts believe these tools will become more crucial in the future. Scientists face both obstacles and opportunities as a result of this transformation, particularly when the approaches are coupled with automation. (Chan et al., 2019).

\subsection{AI for clinical trials}

A clinical trial is a procedure in which freshly manufactured treatments are given to people to test how well they work. This has taken a significant amount of time and money. The success rate, however, is quite low.

As a result, clinical trial automation has proven to be a benefit for AI and the healthcare business. Furthermore, Artificial Intelligence and healthcare assist in the elimination of time- 
consuming data monitoring procedures. Additionally, AI-assisted clinical trials handle large amounts of data and produce very accurate outcomes. The following are some of the most popular Artificial Intelligence in healthcare applications for clinical trials:

\subsubsection{Intelligent clinical trials}

Traditional 'linear and sequential' clinical trials are still the gold standard for ensuring the efficacy and safety of new drugs. The lengthy, tried-and-true method of distinct and defined stages of randomised controlled trials (RCTs) was developed primarily for evaluating massmarket pharmaceuticals and has remained mostly unchanged in recent decades.

Artificial intelligence has the potential to shorten clinical trial cycle durations while also enhancing productivity and clinical development outcomes. This is the third in a series of reports on AI's impact on the biopharma value chain (Lee, 2021) (Angus, 2020).

Biopharma businesses have been able to obtain increasing volumes of scientific and research information from a multitude of sources in recent years, which is referred to as real-world data (RWD). They have, however, frequently lacked the expertise and tools needed to successfully use this data. Applying predictive AI models and advanced analytics to unlock RWD can help researchers better understand diseases, find relevant patients and important investigators, and enable revolutionary clinical study designs (Woo, 2019).

In combination with an efficient digital infrastructure, clinical trial data might be cleansed, aggregated, coded, preserved, and maintained using AI algorithms. Furthermore, improved electronic data capture (EDC) may reduce the impact of human error in data collection while also allowing for smooth system integration (Mayorga-Ruiz et al., 2019).

\subsubsection{Clinical Trial Cooperation and model sharing}

Researchers from several domains are racing to support the reaction to COVID-19 in an exceptional effort of scientific collaboration. Making a worldwide effect with AI tools would necessitate scalable data, model, and code sharing strategies, as well as application adaptation to local settings and cross-border collaboration (Luengo-Oroz et al., 2020).

Data is required for AI applications. At the three application sizes, there are presently dozens of data-sharing efforts centered on COVID-19, spanning the worldwide, national, and local levels. Genetic sequences, genomic analyses, protein structures, patients clinical information, medical imagery, event data, epidemiological information, movement data, social media comments, news stories, and scientific literature are just a few of the resources available. Hyper-fragmentation of data-sharing activities is a problem since it could lead to advancements that are limited to specific projects and communities. The creation and diffusion of new applications could be accelerated by establishing scalable techniques for data, model, and code sharing. Global, open, comprehensive, comparable, and verifiable data-sharing activities will be useful at this stage in connecting and promoting cooperation between various communities and geographies (Lip et al., 2020) (Luengo-Oroz et al., 2020). 
Open science, aided by multi-stakeholder AI collaborations that operate across international borders, can speed up information distribution and capacity building in national health systems (Shaheen, 2021b). The Epidemic Intelligence from Open Sources (EIOS) network, for example, uses open source data to enable early detection, verification, and assessment of public health hazards and threats (Sucharitha \& Chary, 2021). The network of practice for health care intelligence comprises governments, international organizations, and research institutes that collaborate to assess and share information concerning outbreak occurrences in real time under the principle of collaboration rather than competition in early detection. Global standards and database interoperability may facilitate unified reaction and decision-making at the global, national, and local levels, according to epidemiologists. Understanding the epidemiologic characteristics and risk features of various demographics as the pandemic progresses will necessitate taking into account health system resource capacity, public health measures, environmental factors, and COVID-19's societal consequences (Sucharitha \& Chary, 2021).

Aside from data sharing, there are currently few projects that exchange trained AI models for any of the suggested uses. Constraints imposed by unique computational, design, and infrastructural needs; a shortage of documenting; verification and interpretability issues; and legal concerns about confidentiality and intellectual property are among the obstacles to be overcome. Sharing pre-trained and approved AI models could help solutions adapt faster to different situations. Models used to diagnose illness from pictures, forecast patient results, filter misinformation and misinformation depending on propagating patterns through social media, and distill knowledge graphs from massive collections of scholarly papers are instances of algorithms that could be broadly useful (Luengo-Oroz et al., 2020) (Shaheen, 2021a) (Harrer et al., 2019).

\subsection{Patient Care}

Patient outcomes are influenced by artificial intelligence in healthcare. Medical AI firms create a system that aids the patient at every level. Clinical intelligence also analyzes patients' medical data and delivers insights to help them enhance their quality of life. The following are a few significant clinical intelligence systems that improve patient care:

\subsubsection{Maternal Care}

The following is a potential technique for identifying high-risk moms and reducing maternal mortality and problems after childbirth:

a) Predicting whether expectant mothers are at significant risk of difficulties during delivery using electronic health data and artificial intelligence (AI).

2) Using digital technology to increase patient entry to both regular and high-acuity care (i.e., more sophisticated and frequent care) throughout their pregnancy.

When compared to delivering in higher-acuity clinics with more strong resources and clinical experience, high-risk obstetric women who deliver their infants at low-acuity clinics have a higher risk of developing serious maternal morbidity. 


\subsubsection{Healthcare Robotics}

In addition to medical personnel, certain medical robots assist patients. Exoskeleton robots, for example, can assist paralyzed patients in walking again and becoming self-sufficient (Shi et al., 2019). A smart prosthesis is another example of technology in action. These bionic limbs attach sensors that render them more responsive and accurate than natural body parts, with the option of covering them in bionic skin and connecting them to the user's muscles. Robots can help with rehabilitation and surgery. Cyberdyne's Hybrid Assistive Limb (HAL) exoskeleton, for example, is designed to help patients rehabilitate from conditions that lead to lower limb disorders, such as spinal cord injuries and strokes, by using sensors placed on the skin to efficiently detect electrical signals in the patient's body and responding with movement at the joint (Cruciger et al., 2016).

\subsubsection{Genetics AI Data-Driven Medicine}

From genome sequencing to creating a tailored health status from the data in our fitness/activity trackers, today 's healthcare consumer has grown increasingly involved in their personal medical treatment. All of this big data is being compiled and linked to produce a more predictive picture of our health or medical status. Data-driven medicine has the potential to improve not just the precision and agility of genetic disease detection, but also to open the door to individualized medical treatments (Hummel \& Braun, 2020).

\subsubsection{AI-powered Stethoscope}

One notable advantage is that, unlike traditional stethoscopes, the readings may be taken even in noisy environments, allowing for more accurate diagnosis. The records can be obtained by anybody and telemetered to the doctor because there is no need for training to use the digital instrument (Prabu, 2021). This also lowers their chance of contracting COVID-19 and makes it easier to provide better medical care in inaccessible areas and for chronically ill patients. Artificial intelligence (AI) and machine learning have made it possible for computers to discover illness patterns and abnormalities from massive amounts of clinical data. Blood flowing through regular arteries is different from blood that flows around a blood clot in the blood vessels, thus the same idea applies here (J. Agrawal, 2018).

\section{Conclusion}

Artificial intelligence (AI) is progressively being used to healthcare, as it becomes more prevalent in modern enterprise and everyday life. Artificial intelligence has the potential to help healthcare providers in a variety of ways, including patient treatment and administrative tasks. The majority of AI and healthcare innovations are useful in the healthcare industry, but the strategies they assist can be rather different. While some publications on artificial intelligence in health claim that AI can perform just as well as or better than humans at specific processes, such as diagnosing sickness, it will be a long time before AI in healthcare replaces people for a wide range of medical jobs. 
Despite such significant improvements, AI usage in healthcare remains very much in early stages. Continuous research continues to add additional capabilities to the technology, resulting in larger advancements in the coming years across a variety of industries. AI and machine learning have a lot to contribute in the critical healthcare sector, which is undergoing one of the swiftest digital transformations at the moment, and amenities have the possibility to substantially improve quality of life for patients.

\section{References}

Agrawal, J. (2018). Stethee, an AI Powered Electronic Stethoscope. Anaesthesia, Pain \& Intensive Care, 22(3), 412-413.

Agrawal, P. (2018). Artificial intelligence in drug discovery and development. $J$ Pharmacovigil, 6(2).

Angus, D. C. (2020). Randomized clinical trials of artificial intelligence. Jama, 323(11), 1043-1045.

Chan, H. C. S., Shan, H., Dahoun, T., Vogel, H., \& Yuan, S. (2019). Advancing drug discovery via artificial intelligence. Trends in Pharmacological Sciences, 40(8), 592604.

Cruciger, O., Schildhauer, T. A., Meindl, R. C., Tegenthoff, M., Schwenkreis, P., Citak, M., \& Aach, M. (2016). Impact of locomotion training with a neurologic controlled hybrid assistive limb (HAL) exoskeleton on neuropathic pain and health related quality of life (HRQoL) in chronic SCI: a case study. Disability and Rehabilitation: Assistive Technology, 11(6), 529-534.

Díaz, Ó., Dalton, J. A. R., \& Giraldo, J. (2019). Artificial intelligence: a novel approach for drug discovery. Trends in Pharmacological Sciences, 40(8), 550-551.

Greenberg, N., Docherty, M., Gnanapragasam, S., \& Wessely, S. (2020). Managing mental health challenges faced by healthcare workers during covid-19 pandemic. Bmj, 368 .

Habermann, J. (2021). Psychological impacts of COVID-19 and preventive strategies: A review.

Harrer, S., Shah, P., Antony, B., \& Hu, J. (2019). Artificial intelligence for clinical trial design. Trends in Pharmacological Sciences, 40(8), 577-591.

Holzinger, A., Biemann, C., Pattichis, C. S., \& Kell, D. B. (2017). What do we need to build explainable AI systems for the medical domain? ArXiv Preprint ArXiv:1712.09923.

Hummel, P., \& Braun, M. (2020). Just data? Solidarity and justice in data-driven medicine. Life Sciences, Society and Policy, 16(1), 1-18.

Lee, E. (2021). How do we build trust in machine learning models? Available at SSRN 3822437.

Lip, S., Visweswaran, S., \& Padmanabhan, S. (2020). Transforming Clinical Trials with Artificial Intelligence. In Artificial Intelligence (pp. 297-306). Productivity Press.

Luengo-Oroz, M., Pham, K. H., Bullock, J., Kirkpatrick, R., Luccioni, A., Rubel, S., Wachholz, C., Chakchouk, M., Biggs, P., \& Nguyen, T. (2020). Artificial intelligence cooperation to support the global response to COVID-19. Nature Machine Intelligence, 2(6), 295-297.

Maphumulo, W. T., \& Bhengu, B. R. (2019). Challenges of quality improvement in the healthcare of South Africa post-apartheid: A critical review. Curationis, 42(1), 1-9.

Mayorga-Ruiz, I., Jiménez-Pastor, A., Fos-Guarinos, B., López-González, R., García-Castro, F., \& Alberich-Bayarri, Á. (2019). The Role of AI in Clinical Trials. In Artificial 
Intelligence in Medical Imaging (pp. 231-243). Springer.

McNeill, P. M., \& Walton, M. (2002). Medical harm and the consequences of error for doctors. The Medical Journal of Australia, 176(5), 222-225.

Pavli, A., Theodoridou, M., \& Maltezou, H. C. (2021). Post-COVID syndrome: Incidence, clinical spectrum, and challenges for primary healthcare professionals. Archives of Medical Research.

Prabu, A. (2021). SmartScope: An AI-Powered Digital Auscultation Device To Detect Cardiopulmonary Diseases.

Ross, S., Bond, C., Rothnie, H., Thomas, S., \& Macleod, M. J. (2009). What is the scale of prescribing errors committed by junior doctors? A systematic review. British Journal of Clinical Pharmacology, 67(6), 629-640.

Shaheen, M. Y. (2021a). Adoption of machine learning for medical diagnosis.

Shaheen, M. Y. (2021b). AI in Healthcare: medical and socio-economic benefits and challenges.

Shi, D., Zhang, W., Zhang, W., \& Ding, X. (2019). A review on lower limb rehabilitation exoskeleton robots. Chinese Journal of Mechanical Engineering, 32(1), 1-11.

Sucharitha, G., \& Chary, D. V. (2021). Predicting the effect of Covid-19 by using artificial intelligence: A case study. Materials Today: Proceedings.

Ting, D. S. W., Liu, Y., Burlina, P., Xu, X., Bressler, N. M., \& Wong, T. Y. (2018). AI for medical imaging goes deep. Nature Medicine, 24(5), 539-540.

Vaishya, R., Javaid, M., Khan, I. H., \& Haleem, A. (2020). Artificial Intelligence (AI) applications for COVID-19 pandemic. Diabetes \& Metabolic Syndrome: Clinical Research \& Reviews, 14(4), 337-339.

van der Schaar, M., Alaa, A. M., Floto, A., Gimson, A., Scholtes, S., Wood, A., McKinney, E., Jarrett, D., Lio, P., \& Ercole, A. (2021). How artificial intelligence and machine learning can help healthcare systems respond to COVID-19. Machine Learning, 110(1), $1-14$.

Woo, M. (2019). An AI boost for clinical trials. Nature, 573(7775), S100-S100. 